\title{
MRI Findings of Immune Checkpoint Inhibitor-Induced Hypophysitis: Possible Association with Fibrosis
}

(D) Kurokawa, (D) Y. Ota, (D). Gonoi, (D) A. Hagiwara, (D). Kurokawa, (DH. Mori, (D)E. Maeda, (D). Amemiya, (D)Y. Usui, (D) N. Sato, (D). Nakata, (D)T. Moritani, and (D). Abe

\begin{abstract}
BACKGROUND AND PURPOSE: Hypophysitis is one of the well-known adverse effects of immune checkpoint inhibitors. Immune checkpoint inhibitor-induced hypophysitis frequently causes irreversible hypopituitarism, which requires long-term hormone replacement. Despite the high frequency and clinical significance, characteristic MR imaging findings of immune checkpoint inhibitor-induced hypophysitis have not been established. In the present study, we aimed to review and extract the MR imaging features of immune checkpoint inhibitor-induced hypophysitis.
\end{abstract}

MATERIALS AND METHODS: This retrospective international multicenter study comprised 20 patients with melanoma who were being treated with immune checkpoint inhibitors and clinically diagnosed with immune checkpoint inhibitor-induced hypophysitis. Three radiologists evaluated the following MR imaging findings: enlargement of the pituitary gland and stalk; homogeneity of enhancement of the pituitary gland; presence/absence of a well-defined poorly enhanced area and, if present, its location, shape, and signal intensity in T2Wl; and enhancement pattern in contrast-enhanced dynamic MR imaging. Clinical symptoms and hormone levels were also recorded.

RESULTS: Enlargement of the pituitary gland and stalk was observed in 12 and 20 patients, respectively. Nineteen patients showed poorly enhanced lesions (geographic hypoenhancing lesions) in the anterior lobe, and 11 of these lesions showed hypointensity on T2WI. Thyrotropin deficiency and corticotropin deficiency were observed in 19/20 and 12/17 patients, respectively, which persisted in 12/19 and 10/12 patients, respectively, throughout the study period.

CONCLUSIONS: Pituitary geographic hypoenhancing lesions in the anterior lobe of the pituitary gland are characteristic and frequent MR imaging findings of immune checkpoint inhibitor-induced hypophysitis. They reflect fibrosis and are useful in distinguishing immune checkpoint inhibitor-induced hypophysitis from other types of hypophysitis/tumors.

ABBREVIATIONS: CTCAE = Common Terminology Criteria for Adverse Events; CTLA-4 = cytotoxic T-lymphocyte-associated antigen-4; ICI $=$ immune checkpoint inhibitor; ICl-H = immune checkpoint inhibitor-induced hypophysitis; PD-1 = programmed death-1; PD-L1 = programmed death-ligand-1

$\mathbf{R}$ ecent advances in the understanding of tumor immunology have led to the development of immune checkpoint inhibitors (ICIs), such as cytotoxic T-lymphocyte-associated antigen-4

Received April 11, 2020; accepted after revision June 2

From the Department of Radiology (R.K., W.G., E.M., S.A., Y.U., O.A.), Graduate School of Medicine, The University of Tokyo, Tokyo, Japan; Division of Neuroradiology (Y.O., T.M.), Department of Radiology, Michigan Medicine, Ann Arbor, Michigan; Department of Radiology (A.H.), Juntendo University School of Medicine, Tokyo, Japan; Department of Radiology (M.K.), Tokyo Metropolitan Cancer and Infectious Diseases Center Komagome Hospital, Tokyo, Japan; Department of Radiology (H.M.), Jichi Medical University, Tochigi-ken, Japan; Department of Radiology (N.S.), Tokyo Metropolitan Neurological Hospital, Tokyo, Japan; and Department of Radiology (Y.N.), National Center of Neurology and Psychiatry, Tokyo, Japan.

Please address correspondence to Wataru Gonoi, MD, Department of Radiology, Graduate School of Medicine, The University of Tokyo, 7-3-1 Hongo, Bunkyo-ku, Tokyo 113-8655, Japan; e-mail: watapi-tky@umin.net

- Indicates open access to non-subscribers at www.ajnr.org

http://dx.doi.org/10.3174/ajnr.A6692
(CTLA-4) inhibitors, programmed death-1 (PD-1) inhibitors, and programmed death-ligand-1 (PD-L1) inhibitors, which are widely used as breakthrough treatments for a wide variety of malignancies, such as melanoma, pancreatic cancer, and nonsmall-cell lung cancer. ICIs exert their antitumor effects by promoting the attack on activated T-cells in tumors; however, a risk of adverse effects prevails due to the infiltration of activated Tcells into systemic organs, where they may cause an excessive immune response. ${ }^{1}$ Such adverse effects are called immunerelated adverse effects because they present symptoms similar to those of autoimmune diseases, such as skin rash, hypothyroidism, and adrenal insufficiency. Immune-related adverse effects can be expressed in all organs of the body, including the skin, endocrine system, respiratory system, gastrointestinal system, and central nervous system. 
Hypophysitis is an inflammation of the pituitary gland and is one of the typical symptoms of immune-related adverse effects; it results in headache and secondary endocrinopathy, depending on the site and extent of injury due to the pituitary infiltration of activated T-cells. ${ }^{1}$ Hypophysitis caused by the anti-CTLA-4 antibody, ipilimumab (Yervoy; Bristol-Myers Squibb), was first reported in 2003 by Phan et al. ${ }^{2}$ According to a systematic review by Barroso-Sousa et al, ${ }^{3}$ ICI-induced hypophysitis (ICI-H) occurs in association with ICI therapy in the following order of frequency: combination therapy (ipilimumab plus PD-1 inhibitors, $6.4 \%)$, ipilimumab alone (3.2\%), PD-1 inhibitors alone $(0.4 \%)$, and PD-L1 inhibitors alone $(<0.1 \%)$. The fatality rate with ICI-H has been reported to be $2 \%$, and the associated endocrine toxicity is very high $(>91 \%) .{ }^{4,5}$ Endocrinopathy due to ICI-H is often irreversible and requires hormone replacement in $>89 \%$ of patients. ${ }^{4-7}$ Despite its high incidence, knowledge of the histopathologic findings of ICI-H is scarce. The first postmortem case was reported in 2016 by Caturegli et al, ${ }^{5}$ who reported a case of tremelimumab-(an immunoglobulin-2 monoclonal antibody against CTLA-4 produced by AstraZeneca) induced hypophysitis. A postmortem examination revealed near-complete destruction of the anterior lobe of the pituitary gland caused by extensive necrosis and fibrosis and pathologically exhibited lymphocytic and necrotizing hypophysitis. ${ }^{5}$ Contrast MR imaging in the same patient showed a poorly enhanced area in the anterior lobe of the pituitary gland, which was thought to reflect necrosis. ${ }^{5}$ However, there are no comprehensive reports of MR imaging findings specific to ICI-H that summarize the enhancement and enlargement of the pituitary gland and/or stalk, as in other types of hypophysitis. ${ }^{6,8-15}$ Therefore, in the present study, we aimed to review and extract the MR imaging findings specific to ICI-H.

\section{MATERIALS AND METHODS}

This international multicenter retrospective study was approved by the local ethics committees (The University of Tokyo Hospital and Michigan Medicine; IRB 2019310NI), and the requirement for informed consent from study participants was waived due to the retrospective design of the study.

\section{Patients}

Between January 2013 and March 2020, twenty patients with malignant melanoma who received ICIs and were clinically diagnosed with ICI-H in 2 hospitals in Japan and the United States were included. We collected the following clinical and laboratory data of the patients: the symptoms of the patients; the stage of malignant melanoma; ICIs used for treatment; and the levels of corticotropin, cortisol, thyroid-stimulating hormone, free thyroxine, prolactin, luteinizing hormone, follicle-stimulating hormone, testosterone (if male), growth hormone, and insulin-like growth factor 1 . The Common Terminology Criteria for Adverse Events (CTCAE), Version 4.03, were used to assess the severity of adverse effects. The diagnosis of ICI-H was made clinically when $\geq 1$ pituitary hormonal dysfunction, such as hyposecretion of corticotropin or thyroidstimulating hormone, was identified in patients during or just after the treatment with ICIs with or without physical symptoms, such as headache and nausea. Pituitary dysfunction was diagnosed on the basis of the laboratory reference ranges.

\section{MR Imaging Scanning Protocol}

The MR imaging of the pituitary glands was performed using a $1 \mathrm{~T}$, $1.5 \mathrm{~T}$, or $3 \mathrm{~T}$ scanner. The following scans were required for all patients: axial and/or coronal T2-weighted (TR range: 2222$4851 \mathrm{~ms}$; TE range: $80-120 \mathrm{~ms}$; in-plane resolution: $0.41 \times 0.41-$ $0.90 \times 0.90 \mathrm{~mm}^{2}$; section thickness: $2-5 \mathrm{~mm}$ ), axial and/or coronal T1-weighted (TR range: 450-2000 ms; TE range: 9-20 ms; inplane resolution: $0.41 \times 0.41-0.90 \times 0.90 \mathrm{~mm}^{2}$; section thickness: 2-5 mm), contrast-enhanced 3D-T1-weighted (axial, sagittal, or coronal; TR range: 7.2-600 ms; TE range: $2.3-28.3 \mathrm{~ms}$; voxel size: $\left.0.31 \times 0.31 \times 0.8-1 \times 1 \times 1 \mathrm{~mm}^{3}\right)$, and/or contrast-enhanced $2 \mathrm{D}$ T1-weighted (axial, sagittal, and coronal; dynamic or nondynamic; TR range: $450-1800 \mathrm{~ms}$; TE range: $9-15 \mathrm{~ms}$; in-plane resolution: $0.41 \times 0.41-0.90 \times 0.90 \mathrm{~mm}^{2}$; section thickness: $2-5 \mathrm{~mm}$ ). Contrast-enhanced 3D-T1-weighted images were reconstructed in the axial, coronal, and sagittal planes. Contrast-enhanced MR imaging was performed 90 seconds after the administration of intravenous contrast medium. Contrast-enhanced dynamic MR imaging was performed 15, 30, 45, 60, 75, and 90 seconds after the administration of intravenous contrast medium. MR imaging parameters varied among institutions because of the difference in examination protocols used by the institutions.

\section{Image Analysis}

In total, 3 diagnostic radiologists participated in the process. Two of the 3 diagnostic radiologists (with 6 and 8 years of experience in neuroradiology, and the latter being board-certified) separately interpreted all MR images and determined the numeric and nominal values mentioned below. Final numeric values were obtained by averaging the values of the 2 diagnostic radiologists. In case nominal values were interpreted differently by the 2 radiologists, the final decision was made by the third board-certified diagnostic radiologist with 15 years of experience in neuroradiology. The numeric imaging factor evaluated was thickness of the pituitary stalk. Nominal imaging factors evaluated were as follows: the presence or absence of enlargement of the pituitary gland and stalk; homogeneity of enhancement of the pituitary gland (homogeneous or heterogeneous); the presence or absence of a welldefined, poorly enhanced area and, if present, the location (anterior, middle, or posterior; right, center, left, or bilateral), shape (nodular, linear, ring-shaped, or irregular), and signal intensity in T2WI (hyperintensity, isointensity, or hypointensity). For cases with contrast-enhanced MR imaging, the enhancement pattern was categorized into no enhancement, washout, plateau, or gradually enhancing.

Enlargement of the pituitary gland was diagnosed when its height was $\geq 2$ SDs of its reported average in healthy individuals of the same age group-age range, $21-30$ years: $6.6 \pm 1.5 \mathrm{~mm}$ for men, $7.0 \pm 1.9 \mathrm{~mm}$ for women; age range, $31-40$ years: $6.3 \pm 1.4$ $\mathrm{mm}$ and $6.5 \pm 1.7 \mathrm{~mm}$; age range, $41-50$ years: $6.1 \pm 1.5 \mathrm{~mm}$ and $6.4 \pm 1.3 \mathrm{~mm}$; older than 50 years of age: $6.0 \pm 1.6 \mathrm{~mm}$ and $6.7 \pm 1.9 \mathrm{~mm} .{ }^{16}$ During pregnancy or postpartum, independent standards for women were set from $6.0 \pm 0.99 \mathrm{~mm}$ to $8.8 \pm 0.67$ $\mathrm{mm} .{ }^{17}$ Enlargement of the pituitary stalk was diagnosed when the anterolateral diameter of the pituitary insertion of the stalk was $\geq 2$ SDs longer than its reported average in healthy individuals: 
$2.32 \pm 0.39 \mathrm{~mm}^{18}$ All MR images were evaluated in 3D in each case.

\section{Statistical Analysis}

Interreader agreement was assessed using $\kappa$ statistics and was interpreted as poor $(\kappa<0.20)$, fair $(\kappa=0.21-0.40)$, moderate $(\kappa=0.41-0.60)$, $\operatorname{good}(\kappa=0.61-0.80)$, or very good $(\kappa \geq 0.81)$. Statistical analyses were performed using JMP software (Version 14.2.0; SAS Institute).

\section{RESULTS}

Patient characteristics and clinical data are summarized in Tables 1 and 2, respectively. The mean age of the participants was 55.7 years (range, 30-86 years), and half of the patients were men. No female patient was pregnant or in the postpartum state. All patients were treated for stage III or IV melanoma, and the CTCAE grade was 3 in all cases. ICIs used for treatment were as follows: nivolumab (anti-PD-1 monoclonal antibody, Opdivo; Bristol Myers Squibb) + ipilimumab, 11/20 patients (55.0\%); and ipilimumab alone, 9/20 patients (45.0\%). Blood tests revealed endocrinologic abnormalities such as thyrotropin deficiency (19/20 [95.0\%]), corticotropin deficiency 12/17 (70.6\%), and gonadotropin deficiency (9/16 [56.3\%]). After the mean follow-up period of 21.4 months (range, 2-

Table 1: Patient characteristics

\begin{tabular}{lc}
\multicolumn{1}{c}{ Characteristic } & \\
\hline Age (mean) (range) (yr) & $\mathrm{M} / \mathrm{F}$ ( $\mathrm{F}=10: 10$ \\
Sex & 20.4 (1-62) \\
Follow-up period (mean) (range) (mo) & \\
Clinical stage (No.) & 0 \\
SII & 3 \\
III & 17 \\
IV & \\
CTCAE grade (No.) & 0 \\
0-2 & 20 \\
3 & 0 \\
4,5 & \\
ICls (No.) & 11 \\
Ipilimumab + nivolumab & 9 \\
Ipilimumab & \\
Main physical symptoms (No.) & 13 \\
Headache & 3 \\
Fatigue/malaise & 2 \\
Nausea/vomiting & 1 \\
Edema & 1 \\
Numbness & 0 \\
Loss of vision/visual field disorder & 0 \\
Diabetes insipidus & 0 \\
No symptom & \\
\hline
\end{tabular}

62 months), thyrotropin deficiency in 12/19 (63.2\%) and corticotropin deficiency in 10/12 (83.3\%) patients persisted. Hormonal replacement was administered to all patients, and it was continued throughout the course in $17 / 20(85.0 \%)$ patients.

The characteristics of MR imaging findings are summarized in Table 3. Enlargement of the pituitary gland and stalk was observed in $12(60.0 \%)$ and 20 patients (100\%), respectively. Nineteen patients (95.0\%) demonstrated geographic hypoenhancing lesions. All these geographic hypoenhancing lesions were located in the anterior lobes; and 11/19 patients (57.9\%) showed hypointensity, 7 patients (36.8\%) showed iso-intensity, and 1 patient (5.3\%) showed high intensity on T2WI. Contrast dynamic MR imaging was performed in 2 patients, and pituitary geographic hypoenhancing lesions were enhanced gradually, showing a low intensity in T2WI. Representative cases are shown in Figs 1-3. Four patients showed nonenhanced nodules between the anterior and intermediate middle lobes of the pituitary gland, indicating a Rathke cleft cyst, and all these patients had pituitary geographic hypoenhancing lesions in the anterior lobes separate from the cysts. $\mathrm{T} 2{ }^{*}$-weighted images were available for 12 patients with pituitary geographic hypoenhancing lesions, and the blooming effect indicating hemosiderin deposition was not observed in these patients. Interreader agreement was good or very good for each factor $(\kappa=0.64-1$, Table 4$)$. All patients showed an improvement in physical symptoms with immunotherapy, including high-dose prednisone or interleukin-2, and/or brief withdrawal of the ICIs. Previous MR imaging and follow-up MR imaging were performed in 13 and 12 patients, respectively (previous MR imaging: mean, 136 days [range, 53-375 days] before; follow-up MR imaging: mean, 262 days [range, 20-949 days] after the MR imaging when ICI-H was diagnosed). All previous $\mathrm{MR}$ imaging in 13 patients and follow-up MR imaging in 8 patients were performed with contrast material, and the remaining follow-up MR imaging in 4 patients was performed without contrast material. Previous MR imaging showed no abnormal findings, and follow-up MR imaging in all the patients who underwent it confirmed the resolutions of the abnormal findings.

\section{DISCUSSION}

In the present study, we found that the MR imaging of ICI-H demonstrated not only the nonspecific enlargement of pituitary glands and stalks but also the characteristic geographic hypoenhancing lesions in the anterior lobe, and all but 1 patient showed hypo- or isosignal intensities, respectively, in T2WI. These characteristic and frequent $\mathrm{MR}$ imaging findings, along with the

Table 2: Hormone abnormalities

\begin{tabular}{llccc}
\hline & Laboratory Reference Range & $\begin{array}{c}\text { Pituitary Hormone } \\
\text { Abnormalities (\%) }\end{array}$ & $\begin{array}{c}\text { Last Follow-Up, Hormone } \\
\text { Abnormalities (\%) }\end{array}$ & $\begin{array}{c}\text { Hormonal } \\
\text { Recovery (\%) }\end{array}$ \\
\hline Corticotropin deficiency & $\mathrm{ACTH}, 5-63.3 \mathrm{pg} / \mathrm{mL}$ & $12 / 17(70.6)$ & $10 / 12(83.3)$ & $2 / 12(16.7)$ \\
Thyrotropin deficiency & $\mathrm{TSH}, 0.30-5.50 \mu \mathrm{lU} / \mathrm{mL}$ & $19 / 20(95.0)$ & $12 / 19(63.2)$ & $7 / 19(36.8)$ \\
Gonadotropin deficiency & $\mathrm{FSH}, 1.5-10.0 \mathrm{mlU} / \mathrm{mL}(\mathrm{male}) ;$ & $9 / 16(56.3)$ & $1 / 5(20.0)$ & - \\
& $2.0-131 \mathrm{mlU} / \mathrm{mL}(\mathrm{female})^{\mathrm{a}}$ & & & - \\
Diabetes insipidus & Based on symptom & $0 / 20(0)$ & - \\
\hline
\end{tabular}

Note:-ACTH indicates adrenocorticotropic hormone; TSH, thyroid stimulating hormone; FSH, follicle stimulating hormone; mIU, milli-international units. ${ }^{a}$ Depending on age. 
patient being under treatment with ICIs, are useful for the diagnosis of ICI-H.

MR imaging is a recommended technique for diagnosing hypophysitis; particularly, gadolinium-enhanced pituitary MR imaging has been reported to be useful for the diagnosis. ${ }^{7,14}$ However, previous studies have summarized MR imaging findings of ICI-H as moderate to intense, with homogeneous enhancement and enlargement of the pituitary gland with or without enlargement of the pituitary stalk, ${ }^{6,7,14}$ which are nonspecific and common in hypophysitis due to other causes. ${ }^{8-13}$ Pathologic variants of hypophysitis include lymphocytic, granulomatous, immunoglobulin-4related, xanthomatous, and necrotizing.

Table 3: MR imaging findings

\begin{tabular}{lc}
\multicolumn{1}{c}{ Finding } & No. (\%) \\
\hline Enlargement of pituitary gland & $12(60.0)$ \\
Gadolinium-enhanced MR imaging $(n=20)$ & \\
Enlargement of pituitary stalk & $20(100)$ \\
Hypoenhancing lesions in the pituitary gland & $19(95.0)$ \\
High intensity on T2WI & $1 / 19(5.3)$ \\
Isointensity on T2WI & $7 / 19(36.8)$ \\
Low intensity on T2WI & $11 / 19(57.9)$ \\
Shape of hypoenhancing lesion $(n=19)$ & \\
Linear & $8(42.1)$ \\
Nodular & $4(21.1)$ \\
Ring-shaped & $1(5.3)$ \\
Irregular & $6(31.6)$ \\
\hline
\end{tabular}

Characteristic MR imaging findings are not established for ICI-H. ${ }^{7,9}$ Of note, we found pituitary geographic hypoenhancing lesions in all but 1 patient. More than half of these lesions showed a low intensity on T2WI (57.9\%) without blooming effect in all patients with $\mathrm{T} 2{ }^{*}$-weighted imaging, which is suggestive of fibrosis rather than necrosis, cystic degeneration, or hemorrhage as seen in a few patients with lymphocytic hypophysitis, xanthomatous hypophysitis, and necrotizing hypophysitis. ${ }^{19-21}$ Two patients showed a gradual enhancement consistent with the low signal intensity on T2WI, which is also supportive of fibrosis. On the contrary, 1 patient with a pituitary geographic hypoenhancing lesion (5.0\%) showed a high intensity on T2WI, suggesting a cystic change, but taking into consideration the postmortem report of tremelimumab (anti-CTLA4)-induced hypophysitis by Caturegli et al, ${ }^{5}$ it seems to be a necrosis rather than a cystic change. On the basis of the MR imaging findings mentioned above, we speculate that ICI-H has a unique tendency to cause fibrosis and rarely becomes necrotic.

Furthermore, we discuss the distribution and morphology of pituitary geographic hypoenhancing lesions. It is well-established that in patients with ICI-H, the anterior lobe of the pituitary gland is mainly involved, whereas the involvement of the posterior lobe is less frequent. ${ }^{22-24}$ Therefore, in the present study, all pituitary geographic hypoenhancing lesions were localized in the anterior lobe. Although the existence of pituitary geographic hypoenhancing lesions was previously unknown, this MR imaging finding could be retrospectively found in some case reports;

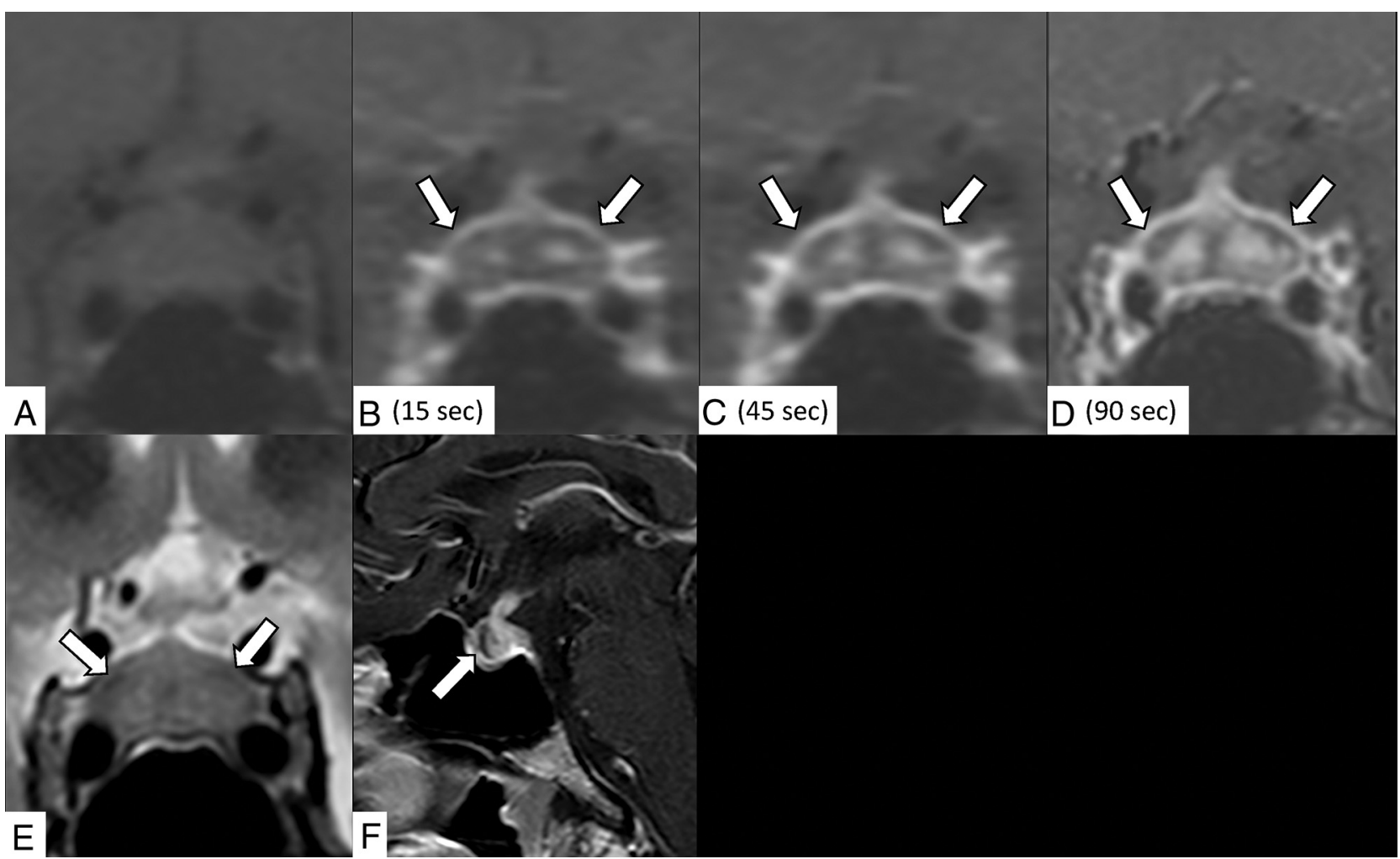

FIG 1. A 31-year-old man receiving ipilimumab for stage IV melanoma with hypothyroidism and headache for a week. Contrast-enhanced coronal dynamic MR imaging demonstrates an enlarged pituitary gland and stalk and a gradually enhanced bilateral ring-shaped hypoenhancing lesion (precontrast $[A] ; 15,45$, and 90 seconds after contrast medium injection $[B-D]$; arrows indicate lesions). The lesion shows mild hypointensity on the T2-weighted coronal image ( $E$, arrows). Contrast-enhanced sagittal MR imaging shows the hypoenhancing lesion in the anterior lobe (F, arrow). 


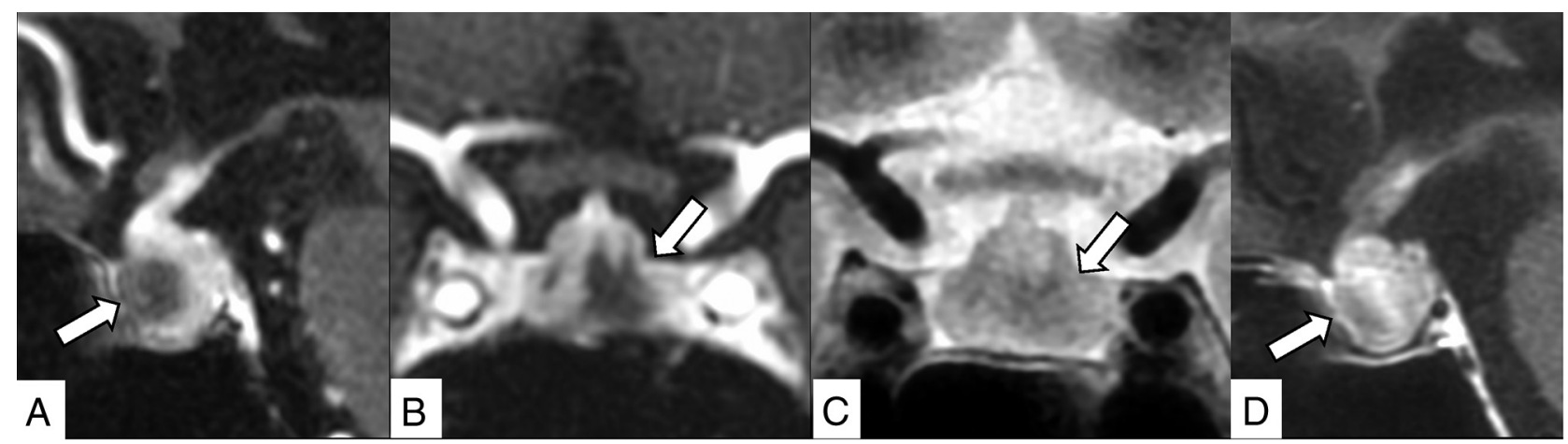

FIG 2. An 82-year-old woman receiving nivolumab plus ipilimumab for stage IV melanoma with corticotropin deficiency, hypothyroidism, and headache for 9 days. Contrast-enhanced 3D-MR imaging ( 90 seconds after contrast medium injection) demonstrates an enlarged pituitary gland and stalk and an irregularly shaped hypoenhancing lesion in the anterior lobe ( $A$, sagittal reconstruction; $B$, coronal reconstruction, arrows). The lesion shows mild hypointensity on T2WI (C, arrow). The lesion is gradually enhanced in contrast-enhanced 2D sagittal MR imaging performed 90 more seconds after the 3D MR imaging ( $D$, arrow).

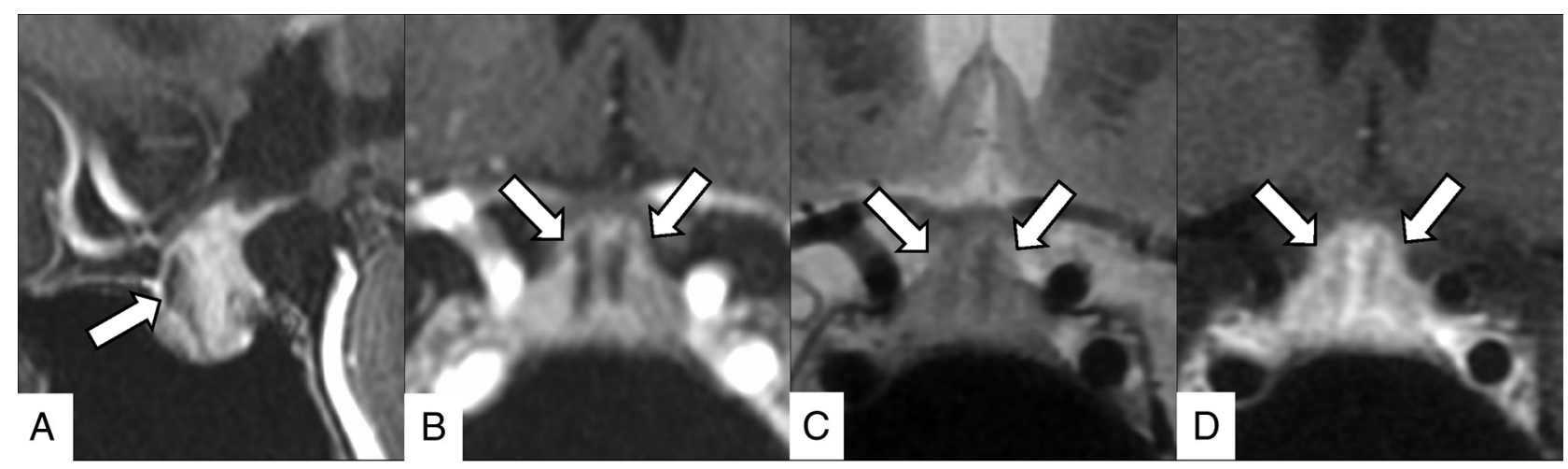

FIG 3. A 40-year-old woman receiving nivolumab plus ipilimumab for stage IV melanoma with corticotropin deficiency, hypothyroidism, and hand edema for 10 days. Contrast-enhanced 3D-MR imaging ( 90 seconds after contrast medium injection) demonstrates an enlarged pituitary gland and stalk and bilateral linear hypoenhancing lesions ( $A$, sagittal reconstruction; $B$, coronal reconstruction, arrows). These lesions show hypointensity on the T2-weighted coronal image ( $C$, arrows), and gradual enhancement is demonstrated in contrast-enhanced 2D coronal MR imaging performed 90 more seconds after the 3D MR imaging ( $D$, arrows).

Table 4: Interrater reliability between 2 raters

\begin{tabular}{lc}
\multicolumn{1}{c}{ MR Imaging Finding } & \multicolumn{1}{c}{$\boldsymbol{\kappa}$} \\
\hline Pituitary height & 0.8326 \\
Stalk thickness & 0.9136 \\
Homogeneity of enhancement & 1 \\
Hypoenhancing lesion & $0.6429^{\mathrm{a}}$ \\
Shape & 0.8535 \\
Signal intensity in T2WI & 0.8077 \\
Location (AP) & 1 \\
Location (RL) & 0.8462 \\
Pattern of enhancement & 1 \\
\hline
\end{tabular}

Note:-AP indicates anterior-posterior; RL, right-left.

${ }^{a}$ The difference in judgment between the 2 raters was observed only in 1 case (rater 1, 19/20 versus rater $2,18 / 20$ ); this was subsequently arbitrated by a third radiologist as positive for a hypoenhancing lesion.

for example, 1 of the 3 patients in the report of Carpenter et al, ${ }^{15}$ 1 patient in the study of Albarel et al, ${ }^{14}$ and 1 patient in the study of Caturegli et $\mathrm{al}^{5}$ demonstrated pituitary geographic hypoenhancing lesions in the anterior lobe of the pituitary gland. The pituitary geographic hypoenhancing lesions showed a variety of shapes, probably due to differences in the distribution of fibrosis and necrosis in each patient. In terms of endocrinologic prognosis, prolonged hormonal replacement is required for patients with ICI-H in general, especially for corticotropin deficiency, and multidisciplinary follow-up should be performed. ${ }^{14}$ According to the review by Albarel et al, ${ }^{14}$ in 2019, corticotropin deficiency and thyrotropin deficiency persisted in 63/72 (84.9\%) and 34/75 (45.3\%) patients, respectively, during the last follow-up; and long-term hormone replacement was needed in patients with ICI-H. These findings are consistent with the findings of this study, in which corticotropin deficiency and thyrotropin deficiency persisted in 10/12 (83.3\%) and 12/19 (63.2\%) patients, respectively, throughout the study period. The high frequency of anterior hypopituitarism and the rarity of diabetes insipidus seem to correlate with the high frequency of anterior lobe involvement in ICI-H.

In the present study, ipilimumab was used in all patients, and it was given as a combination therapy with nivolumab in $10 / 19$ patients (52.6\%). Immune-related adverse effects are caused by injury to the target organ by a combination of autoreactive T-cells, autoantibodies, and/or proinflammatory cytokines, but the frequency and extent of adverse reactions varied between drugs. ${ }^{1}$ ICI-H is observed more frequently (3.2\%) with CTLA-4 inhibitors than other ICIs, and the expression of CTLA-4 on normal pituitary 
cells is expected to be the underlying cause. ${ }^{3,25}$ The frequency of PD-1 inhibitor-induced hypophysitis is low at $0.4 \%$, whereas the frequency of hypophysitis caused by combination therapy (ipilimumab plus PD-1 inhibitors) is higher at $6.4 \% .^{3}$ Furthermore, hypophysitis associated with combination therapy or ipilimumab alone is more frequently high-grade $(\geq 3)$ in CTCAE than with PD-1/PD-L1 inhibitors. ${ }^{26,27}$ Consistently, the CTCAE grade was 3 for all patients in the present study.

According to the review by Larkin et $\mathrm{al}^{27}$ the frequency of highgrade-versus-low-grade ICI-H was $1.0 \%$ (3/313) versus $6.1 \%$ (19/ 313) for combination therapy (nivolumab plus ipilimumab) and $1.9 \%(6 / 311)$ versus $1.9 \%(6 / 311)$ for ipilimumab alone. Thus, it is likely that more than an equal number of patients with potential low-grade (CTCAE grade $\leq 2$ ) hypophysitis existed during the current patient enrollment period but were not diagnosed because their conditions were clinically and endocrinologically mild. Further studies on the MR imaging findings of ICI-H in patients with low-grade hypophysitis are needed in the future.

This study had the following limitations: First, histologic confirmation of the pituitary gland was not performed. However, the possibility of other types of hypophysitis or tumors is low, because all patients developed physical symptoms during or immediately after the immunotherapy with ICIs, and the symptoms improved with additional immunotherapy or withdrawal of ICIs. In addition, none of the patients with previous MR imaging showed abnormal findings in the pituitary gland, and all patients with follow-up MR imaging showed the resolution of the abnormal findings. Second, the frequency of pituitary geographic hypoenhancing lesions in hypophysitis due to other causes was not examined. To the best of our knowledge, there are no reports in the literature of a high incidence of poorly enhanced areas and fibrosis in hypophysitis due to other causes. Third, patients with low-grade ICI-H with a CTCAE grade of $\leq 2$ were not included in the present study. Therefore, the frequency of MR imaging findings, such as pituitary geographic hypoenhancing lesions, might be different in patients with lowgrade ICI-H. Finally, all patients included in the present study were administrated ipilimumab-containing therapies, but one case series including 4 patients have reported that patients with PD-1/ PD-L1 blockade-induced hypophysitis did not show any abnormalities in MR imaging. ${ }^{28}$ To clarify whether "pituitary geographic hypoenhancing lesions" are unique to ipilimumab, further studies investigating ICI-H associated with other ICI therapies are needed.

\section{CONCLUSIONS}

Contrast MR imaging of ICI-H frequently demonstrates T2weighted low-intensity pituitary geographic hypoenhancing lesions in the anterior lobe of the pituitary gland. These characteristic and frequent MR imaging findings may reflect fibrosis and are useful in distinguishing ICI-H from other types of hypophysitis and tumors.

\section{ACKNOWLEDGMENTS}

We thank Dr Naohiro Makise, Department of Pathology, The University of Tokyo Hospital, for his advice on histopathology.

Disclosures: Osamu Abe_UNRELATED: Grants/Grants Pending: Bayer Yakuhin, Ltd, Canon Medical Systems, Eisai Co Ltd, FUJIFILM Toyama Chemical Co Ltd, GE Healthcare, Guerbet Japan, Philips Healthcare, Siemens Healthcare Diagnostics KK,
Daiichi Sankyo Company Ltd, Fuji Pharma Co Ltd, and Nihon Medi-Physics Co Ltd*; Payment for Lectures Including Service on Speakers Bureaus: Bayer Yakuhin Ltd, Canon Medical Systems, Eisai Co Ltd, FUJIFILM Toyama Chemical Co Ltd, GE Healthcare, Guerbet Japan, Philips Healthcare, and Siemens Healthcare Diagnostics KK. *Money paid to the institution.

\section{REFERENCES}

1. Thompson JA, Schneider BJ, Brahmer J, et al. Management of immunotherapy-related toxicities, version 1. 2019. J Natl Compr Canc Netw 2019;17:255-89 CrossRef Medline

2. Phan GQ, Yang JC, Sherry RM, et al. Cancer regression and autoimmunity induced by cytotoxic $\mathrm{T}$ lymphocyte-associated antigen 4 blockade in patients with metastatic melanoma. Proc Natl Acad Sci USA 2003;100:8372-77 CrossRef Medline

3. Barroso-Sousa R, Barry WT, Garrido-Castro AC, et al. Incidence of endocrine dysfunction following the use of different immune checkpoint inhibitor regimens: a systematic review and meta-analysis. JAMA Oncol 2018;4:173-82 CrossRef Medline

4. Wang DY, Salem JE, Cohen JV, et al. Fatal toxic effects associated with immune checkpoint inhibitors: a systematic review and metaanalysis. JAMA Oncol 2018;4:1721-88 CrossRef Medline

5. Caturegli P, Di Dalmazi G, Lombardi M, et al. Hypophysitis secondary to cytotoxic T-lymphocyte-associated protein $\mathbf{4}$ blockade: insights into pathogenesis from an autopsy series. Am J Pathol 2016;186:322535 CrossRef Medline

6. Tan MH, Iyengar R, Mizokami-Stout K, et al. Spectrum of immune checkpoint inhibitors-induced endocrinopathies in cancer patients: a scoping review of case reports. Clin Diabetes Endocrinol 2019;5:1 CrossRef Medline

7. Joshi MN, Whitelaw BC, Carroll PV. Mechanisms in endocrinology: hypophysitis: diagnosis and treatment. Eur J Endocrinol 2018;179: R151-63 CrossRef Medline

8. Nakata Y, Sato N, Masumoto T, et al. Parasellar T2 dark sign on MR imaging in patients with lymphocytic hypophysitis. AJNR Am J Neuroradiol 2010;31:1944-50 CrossRef Medline

9. Bando H, Iguchi G, Fukuoka H, et al. The prevalence of IgG4related hypophysitis in 170 consecutive patients with hypopituitarism and/or central diabetes insipidus and review of the literature. Eur J Endocrinol 2014;170:161-72 CrossRef Medline

10. Iseda I, Hida K, Tone A, et al. Prednisolone markedly reduced serum IgG4 levels along with the improvement of pituitary mass and anterior pituitary function in a patient with IgG4-related infundibulo-hypophysitis. Endocr J 2014;61:195-203 CrossRef Medline

11. Kong X, Wang R, Yang Y, et al. Idiopathic granulomatous hypophysitis mimicking pituitary abscess. Medicine (Baltimore) 2015;94: e1099 CrossRef Medline

12. Unlu E, Puyan FO, Bilgi S, et al. Granulomatous hypophysitis: presentation and MRI appearance. J Clin Neurosci 2006;13:1062-66 CrossRef Medline

13. Gutenberg A, Caturegli P, Metz I, et al. Necrotizing infundibulohypophysitis: an entity too rare to be true? Pituitary 2012;15:20208 CrossRef Medline

14. Albarel F, Castinetti F, Brue T. Management of endocrine disease: immune check point inhibitors-induced hypophysitis. Eur J Endocrinol 2019;181:R107-18 CrossRef Medline

15. Carpenter KJ, Murtagh RD, Lilienfeld $\mathrm{H}$, et al. Ipilimumabinduced hypophysitis: MR imaging findings. AJNR $A m J$ Neuroradiol 2009;30:1751-53 CrossRef Medline

16. Yadav P, Singhal S, Chauhan S, et al. MRI evaluation of size and shape of normal pituitary gland: age and sex related changes. J Clin Diagn Res 2017;11:TC01-04 CrossRef

17. Dinç, H, Esen F, Demirci A, et al. Pituitary dimensions and volume measurements in pregnancy and postpartum MR assessment. Acta Radiol 1998;39:64-69 CrossRef Medline

18. Satogami N, Miki Y, Koyama T, et al. Normal pituitary stalk: highresolution MR imaging at 3T. AJNR Am J Neuroradiol 2010;31:35559 CrossRef Medline 
19. Lee SJ, Yoo HJ, Park SW, et al. A case of cystic lymphocytic hypophysitis with cacosmia and hypopituitarism. Endocr J 2004;51:37580 CrossRef Medline

20. Hanna B, Li YM, Beutler T, et al. Xanthomatous hypophysitis. J Clin Neurosci 2015;22:1091-97 CrossRef Medline

21. Ćaćić M, Marinković J, Kruljac I, et al. Ischemic pituitary apoplexy, hypopituitarism and diabetes insipidus: a triad unique to necrotizing hypophysitis. Acta Clin Croat 2018;57:768-71 CrossRef Medline

22. Dillard T, Yedinak CG, Alumkal J, et al. Anti-CTLA-4 antibody therapy associated autoimmune hypophysitis: serious immune related adverse events across a spectrum of cancer subtypes. Pituitary 2010;13:29-38 CrossRef Medline

23. Nallapaneni NN, Mourya R, Bhatt VR, et al. Ipilimumab-induced hypophysitis and uveitis in a patient with metastatic melanoma and a history of ipilimumab-induced skin rash. J Natl Compr Canc Netw 2014;12:1077-81 CrossRef Medline
24. Zhao C, Tella SH, Del Rivero J, et al. Anti-PD-L1 treatment induced central diabetes insipidus. J Clin Endocrinol Metab 2018;103:365-69 CrossRef Medline

25. Postow MA, Sidlow R, Hellmann MD. Immune-related adverse events associated with immune checkpoint blockade. $N$ Engl J Med 2018;378:158-68 CrossRef Medline

26. Robert C, Schachter J, Long GV, et al. Pembrolizumab versus ipilimumab in advanced melanoma. $N$ Engl J Med 2015;372:2521-32 CrossRef

27. Larkin J, Chiarion-Sileni V, Gonzalez R, et al. Combined nivolumab and ipilimumab or monotherapy in untreated melanoma. $N$ Engl $J$ Med 2015;373:23-34 CrossRef Medline

28. Lupi I, Brancatella A, Cosottini $M$, et al. Clinical heterogeneity of hypophysitis secondary to PD-1/PD-L1 blockade: insights from four cases. Endocrinol Diabetes Metab Case Rep 2019;2019:19-102 CrossRef Medline 